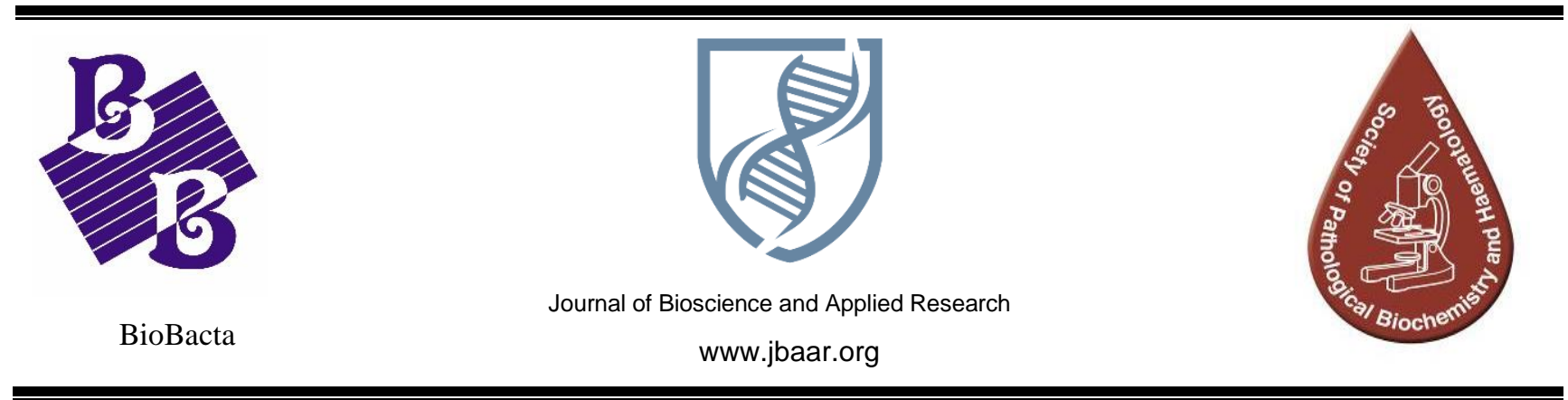

\title{
Evaluation of circulating antigen in urine technique for diagnosis of Schistosoma haematobium infection
}

\author{
Ahmed Abdelhalim Yameny \\ Society of Pathological Biochemistry and Hematology, Egypt \\ Ahmed A. Yameny (Email: dr.ahmedyameny@yahoo.com)
}

\section{ABSTRACT}

DOI: 10.21608/jbaar.2018.155494

Background: Diagnosis of urinary and intestinal schistosomiasis by detecting circulating Schistosome antigens in serum and urine have been developed and assessed to dissolve problems with parasitological diagnosis in low endemic areas or for early diagnosis, this study aimed to evaluate commercially available kit, using circulating antigen in urine as an alternative for microscopic testing of urine to diagnose S.haematobium infection Subjects and methods: This study is a cross-sectional study designed to evaluate circulating antigen a commercially available kit was used for determining urinary schistosomiasis in the study population, this performed on 50 positive S.haematobium samples and 50 negative samples for individuals matching in age and gender, besides, urine microscopic examination was done for detection of S.haematobium eggs by sedimentation centrifugation and nuclepore filtration techniques. Results: The results showed that circulating antigen kit in urine had a sensitivity of 56\% and specificity of $76 \%$. PPVs were $70 \%$ whereas NPVs were $63.3 \%$. As for diagnostic efficiency, it was $66 \%$, where the area under the curve (AUC) was sufficient 0.63. Conclusion and Recommendations: Years ago this commercial kit used in this study and all medical laboratories in Egypt for detection of schistosome antigen in urine is called schistofast bilharzial antigen in urine, this study showed low sensitivity (56\%) and low specificity (76) than expected results for detection of circulating antigen and they are generally more expensive than microscopic examination.

Keywords: Schistosoma haematobium, CAg, circulating antigen, validity, urinary schistosomiasis.

\section{Introduction}

Diagnosis of schistosomiasis is usually performed by parasitological (microscopic detection of eggs), and/or immunological methods (antibody and antigen detection) ${ }^{(1)}$. Methods have been developed for the diagnosis of light infections, which developed on either detection of antibodies specific to schistosome antigens or the presence of schistosome circulating antigens (SCA) in patients (Salah et al., 2006) ${ }^{(2)}$. Schistosome antigens are present in the serum and urine of infected subjects ${ }^{(3)}$. According to their migratory behavior in immunoelectrophoresis, they are 
commonly referred to as circulating anodic antigens (CAA) and circulating cathodic antigens (CCA). These two circulating adult worm antigens are the basis of antigen capture immunoassays ${ }^{(4)}$. Somatic schistosome antigens, such as circulating anodic antigen and circulating cathodic antigen, can be detected and quantified with labeled monoclonal antibodies in serum or urine of infected individuals ${ }^{(5)}$, many attempts have been made to identify the egg antigens which are responsible for inducing those reactions and which proved also to be useful immunodiagnostic reagents (McManus and Loukas, 2008) ${ }^{(6)}$, These tests can differentiate between past and active infections, as the circulating antigens are probably present only when there is active infection (Doenhoff 2004) (7), $^{(7)}$ circulating antigens are released from living worms, antigen levels may correlate directly with parasite load, whilst microscopy does not. This may make the circulating antigen test useful in monitoring the dynamics of worm burdens and clearance of worms after treatment (Cavalcanti 2013; Rollinson 2013) ${ }^{(8,9)}$.

during the acute phase or in recently reinfected cases of schistosomiasis, immature worms may produce worm antigens(e.g., CCA) before eggs are excreted, this process may result in a positive result using the circulating antigen assay and a negative result using a microscopic technique for eggs ${ }^{(10,11)}$. The level of antigen correlates well with the intensity of infection and is rapidly cleared from the circulation following successful treatment ${ }^{(12,13)}$. Feldmeier and others measured CAA and CCA levels four months post-treatment and their results showed a significant decrease in serum CAA and CCA after treatment with praziquantel, but they related this decrease to infection with S.mansoni and not to S.haematobium ${ }^{(14)}$. The main advantage of antigen detection, and particularly CAA detection in serum, is the fact that antigen levels show little fluctuation. A one-point determination, therefore, provides more reliable quantitative data than in the case of a parasitological diagnosis ${ }^{(15)}$. On the other hand, the main disadvantages of antigen detection are related to the availability and cost of the reagents, and to relatively timeconsuming and expensive (ELISA) assay, which also is not suitable to use outside a laboratory setting ${ }^{(15)}$.

Questionnaire and chemical reagent strip for haematuria and proteinuria can be considered for the diagnosis of $S$. haematobium where microscopy is unavailable In areas with a high prevalence of infection $^{(16,17)}$, recently Schistosomiasis infection was decreased in many countries and has been eliminated in Iran, Lebanon, Morocco and Tunisia with an absence of newly recorded cases in the past few years (WHO, 2007) ${ }^{(18)}$. In Egypt (2016), due to different control measures, the overall prevalence S.haematobium and S.mansoni fell to less than $0.2 \%$, and Egypt has started a campaign to reach the final elimination of schistosomiasis by $2020^{(19)}$. So many medical laboratories in Egypt using a commercially available kit by circulating antigen (Schistofast ABC Diagnostic), this study aimed to evaluate commercially available kit, using circulating antigen in urine as an alternative for microscopic testing of urine to diagnose S.haematobium infection.

\section{Material and methods}

\subsection{Study population and ethical consideration}

This study included 100 patients attending Ministry of health laboratory centers, in El-Fayoum Governorate, these study subjects were randomly selected irrespective of the age-group and both genders were included. All the studied population was informed about the purpose of sample collection and their consent was obtained. Patients were free to refuse sample collection.

\subsection{Study design}

This research is a cross-sectional study designed to evaluate circulating antigens by a commercially available kit (Schistofast ABC Diagnostic, New Damietta city ARE) to determine urinary schistosomiasis in the study population. This study 
was targeting customers who came to laboratories of health centers for urine analysis. This was performed on 50 positive S.haematobium samples and 50 negative samples for individuals matching in age and sex.

\subsection{Collection and processing of urine samples}

Clean specimen bottles were labeled with the needed information and issued to the participating individuals whose informed consent was sought earlier, each patient was given a wide mouth screwcapped container into which to void urine. This was carried out between $10.00 \mathrm{am}$ and $2.00 \mathrm{pm}$ when the ova count of S.haematobium is expected to be at its peak ${ }^{(20)}$. In this study, urinary schistosomiasis was defined as the presence of ova of S.haematobium in the urine.

\subsection{Urine microscopy}

Urine samples were examined for the presence of S.haematobium eggs as in the sedimentation method of Cheesbrough (2006) ${ }^{(21)}$. Each urine sample was mixed thoroughly with a glass rod and three samples were taken each $10 \mathrm{ml}$ urine, one sample for sedimentation centrifugation, the other $10 \mathrm{ml}$ urine sample for Nuclepore membrane filteration technique, and the third sample for circulating antigen in urine detection. The first 10 $\mathrm{ml}$ transferred into a centrifuge tube and centrifuged at $2000 \mathrm{rpm}$ for 5 minutes at room temperature. The supernatant was then discarded and sediment was transferred to a microscope glass slide and covered with a coverslip. A drop of Lugol's Iodine was added onto the coverslip before the examination. Examination of the entire sediment was carried out using $\times 10$ objective of a compound light microscope.

The second $10 \mathrm{ml}$ urine sample was examined using the Nuclepore membrane filteration technique for S.haematobium eggs detection as in the method of Cheesbrough (2009) ${ }^{(22)}$. The third urine sample was examined using a commercially circulating antigen kit according to the assay procedures.

\subsection{Assay procedure:}

For 100 urine samples, were tested for the presence of circulating antigen by a commercially available kit (Schistofast ABC Diagnostic, New Damietta city ARE). Which were the same kit used by medical laboratories in Egypt), these steps according to the company method.

Note: 1 - the urine sample was centrifuged (2000 rpm for $5 \mathrm{~min}$.) to remove any turbidity e.g.pus cells, RBCs, -----etc. If centrifuge was not available, urine was left for about $30 \mathrm{~min}$. the supernatant was used.

2- To test the positive and the negative controls (precoated Devices), the assay procedure was followed up except step no. 1 (the addition of urine sample).

* the membrane surface of the test cartilage washed by adding 3 drops of solution(A)(wash solution), drops were allowed for complete absorption.

1- Three drops or (200 ul) of urine was added (supernatant), using a new plastic pipette/sample

2- solution (B), blocking reagent, shake gently, 2 drops were added, drops were allowed to be completely absorbed

3- solution (C), specific MoAb solution, shaken gently, 2 drops were added, drops were allowed to be completely absorbed

*Washed by addition of 3 drops of solution (A), the drops allowed for completely absorbed.

4- solution (D), alkaline phosphatase conjugate, shake gently, 2 drops were added, drops allowed to be completely absorbed.

* Washed by addition of 3 drops of solution (A), the drops allowed for complete absorption. 
5- Two drops or (100 ul) of solution (E), substrate solution, were added using a new plastic pipette, and waited for 2 minutes.

6- Two drops of solution(F), stopper solution, were added, allowed to be completely absorbed, and the result was taken.

\section{Result and report:}

No color Negative bilharzial antigen.

Violet color -Positive bilharzial antigen.

Resultsiolet color intensity may be weak or strong according to the concentration of the bilharzial antigen in the urine sample.

\subsection{Stool microscopy:}

Stool samples were examined for the presence of S.mansoni eggs, only negative stool samples for S.mansoni eggs of 100 population study samples were taken for that research study.

Results collected, coded, tabulated, and analyzed through computer facilities using statistical methods S.haematobium infection was defined as any number of eggs greater than zero found in 10 $\mathrm{ml}$ of urine, was performed to compare with circulating antigen by commercially kit for Diagnosis of Schistosoma haematobium Infection. data analysis Correlation of the circulating antigen results with the gold-standard parasitological data was done using diagnostic accuracy tests. Sensitivity, specificity, positive and negative predictive values (PPV and NPV), likelihood ratios for positive results (LR+) and diagnostic efficiency, and the area under the curve (AUC) were calculated. In this study, we considered using the sum of Nuclepore membranes filteration technique and Centrifugation sedimentation technique results as a gold standard to compare them with circulating antigen by commercially kit (23)

Table (1): shows a diagnostic performance of circulating antigen in urine $(\mathrm{CAg})$ by a commercially available kit (Schistofast ABC Diagnostic) as a diagnostic method for Schistosoma haematobium infection compared to microscopic examination techniques as the gold standard. In this study, we considered using the sum of Nuclepore membrane as filteration technique and Centrifugation sedimentation technique results as a gold standard $^{(23)}$.

The results showed that circulating antigen in urine $(\mathrm{CAg})$ by a commercially available kit had a sensitivity of $56 \%$ and specificity of $76 \%$. PPVs were $70 \%$ whereas NPVs were $63.3 \%$. As for diagnostic efficiency, it was (66\%), where the area under the curve (AUC) was sufficient (0.6). 
Table (1): Shows the percentage of S.haematobium infection using a circulating antigen in urine (CAg) as a diagnostic method for schistosoma haematobium compared to microscopic examination techniques as the gold standard.

\begin{tabular}{|c|c|c|c|c|c|c|c|c|c|c|c|c|}
\hline \multicolumn{2}{|l|}{ Test } & \multicolumn{2}{|c|}{$\begin{array}{l}\text { Microscopic } \\
\text { techniques }\end{array}$} & \multirow{2}{*}{$\begin{array}{c}\text { Total } \\
\text { No. }\end{array}$} & \multirow{2}{*}{$\begin{array}{c}\text { Sensitivity } \\
\%\end{array}$} & \multirow{2}{*}{$\begin{array}{c}\text { Specificity } \\
\%\end{array}$} & \multirow{2}{*}{$\begin{array}{c}\text { PPV } \\
\%\end{array}$} & \multirow{2}{*}{$\begin{array}{c}\text { NPV } \\
\%\end{array}$} & \multirow{2}{*}{$\begin{array}{c}\text { PLR } \\
\%\end{array}$} & \multirow{2}{*}{$\begin{array}{c}\text { NLR } \\
\%\end{array}$} & \multirow{2}{*}{$\begin{array}{c}\text { Diagnostic } \\
\text { Efficiency } \\
\%\end{array}$} & \multirow[t]{2}{*}{ AUC } \\
\hline \multicolumn{2}{|l|}{ Test } & No. & & & & & & & & & & \\
\hline \multirow{3}{*}{$\begin{array}{c}\text { Circulating } \\
\text { antigen in } \\
\text { urine } \\
(\mathrm{CAg})\end{array}$} & No & 38 & 22 & 60 & \multirow{3}{*}{56} & \multirow{3}{*}{76} & \multirow{3}{*}{70} & \multirow{3}{*}{63.3} & \multirow{3}{*}{2.33} & \multirow{3}{*}{0.58} & \multirow{3}{*}{66} & \multirow{3}{*}{0.63} \\
\hline & Yes & 12 & 28 & 40 & & & & & & & & \\
\hline & Total & 50 & 50 & 100 & & & & & & & & \\
\hline
\end{tabular}

\section{Discussion}

Detection of circulating schistosome antigens secreted by live schistosomes in body fluids with specific monoclonal antibodies (MAbs) is a promising approach to the detection of active infection and the assessment of treatment efficacy and effectiveness of future vaccines ${ }^{(24,25)}$.

During schistosome infection, many of the eggs laid by the female worms become trapped in the tissues. The liver is particularly affected in $S$. mansoni and $S$. joponicum infections, while the bladder and ureters are the main organs of egg deposition by $S$. haematobium worms. As the major factor in the pathogenesis of schistosomiasis is the host granulomatous response to antigens secreted from the trapped eggs in host tissues (Pearce, 2005) $^{(26)}$. Furthermore, early diagnosis is not possible because eggs are not found in feces and urine until flukes reach maturity (Armour et al., 1997) ${ }^{(27)}$. Therefore, several Immunodiagnostic methods have been developed for the diagnosis of light infections.

Years ago this commercial kit which was used in this study also used in all medical laboratories in Egypt for detection of Schistosoma antigen in urine is called schisto-fast bilharzial antigen in urine manufactured by ABC diagnostic, Damietta, Egypt. This showed that the sensitivity of circulating antigen in urine was $56 \%$, where the specificity was $76 \%$ and positive predictive value $70 \%$ and kappa coefficient was 0.32 (fair), As for diagnostic efficiency it was $(66 \%)$, where the area under the curve (AUC) was sufficient (0.6), while many of the assays based on antigen detection displays both high specificities and high sensitivities ${ }^{(1)}$.

Zienab A, et al., (1995) detected circulating Schistosome antigen (CSA) in $97 \%$ of urine samples of $S$. mansoni infected school children, CSA was detectable in $100 \%$ of urine samples of mixed S. mansoni and S.haematobium infected patients. They also found the specificity of the CSA reached $98 \%{ }^{(28)}$.

Ndhlovu P, et al., (1996) evaluated circulating anodic antigen (CAA) levels in different age groups in a Zimbabwean between rural community endemic for schistosoma haematobium. They found that specificity was $100 \%$ and the overall sensitivity was $97 \% .^{(29)}$

Mahfouz A, et al., (2012) evaluated different immunological techniques for the diagnosis of schistosoma haematobium in Egypt through detecting soluble egg antigen (SEA) in urine by different methods (latex agglutination technique LAT, sandwich ELISA, and dot-ELISA) They found the sensitivity of circulating antigen in urine ranged from $88.66 \%$ to $94.66 \%$, while the specificity ranged from $91.25 \%$ to $96.25 \%{ }^{(30)}$.

Circulating Schistosome antigen(CSA) disappears rapidly after treatment and can therefore 
be used for assessment of cure. However, the sensitivity of antigen detection varies from $55 \%$ to $100 \%$, being low in low endemic areas with no advantage over stool and urine examination ${ }^{(1)}$.

\section{Conflict of interest}

There are no conflicts of interest.

\section{Financial support and sponsorship}

This research did not receive any specific grant from funding agencies in the public, commercial, or not-for-profit sectors.

\section{References:}

1. Van Lieshout, L., A. M. Polderman, and A. M. Deelder. 2000. Immunodiagnosis of schistosomiasis by determination of the circulating antigens $\mathrm{CAA}$ and $\mathrm{CCA}$, in particular in individuals with recent or light infections. Acta Trop. 77:69-80.

2. Salah, F., A. El Bassiouny, I. Rabie, Z. Demerdash, M. Roshdy, and Z. Shaker, 2006. Human schistosomiasis haematobium: Effective diagnosis of active infection using a pair of monoclonal antibodies against soluble egg antigen. Parasitol. Res., 99: 528-533.

3. Deelder AM, Klappe HT, van den Aardweg GJ, van Meerbeke EH (1976) Schistosoma mansoni: demonstration of two circulating antigens in infected hamsters. Exp Parasitol 40: 189-197.

4. Al-Sherbiny MM, Osman AM, Hancock K, Deelder AM, Tsang VC (1999) Application of immunodiagnostic assays: detection of antibodies and circulating antigens in human schistosomiasis and correlation with clinical findings. Am J Trop Med Hyg 60: 960-966.

5. Deelder AM, Qian ZL, Kremsner PG, et al. Quantitative diagnosis of Schistosoma infections by measurement of circulating antigens in serum and urine. Trop Geogr Med 1994; 46: 233-38.

6. McManus, D.P. and A. Loukas, 2008. Current status of vaccines for schistosomiasis. Clin. Microbiol. Rev., 21: 225-242.
7. Doenhoff MJ, Chiodini PL, Hamilton JV. [Specific and sensitive diagnosis of schistosome infection: can it be done with antibodies?]. Trends in Parasitology 2004;20 (1):35-9.

8. Cavalcanti MG, Silva LF, Peralta RH, Barreto MG, Peralta JM. [Schistosomiasis in areas of low endemicity: a new era in diagnosis]. Trends in Parasitology 2013;29 (2):75-82.

9. Rollinson D, Knopp S, Levitz S, Stothard JR, Tchuem Tchuente LA, Garba A, et al. [Time to set the agenda for schistosomiasis elimination]. Acta Tropica 2013;128 (2): 423-40.

10. Lambertucci JR. Acute schistosomiasis mansoni: revisited and reconsidered. Mem Inst Oswaldo Cruz. 2010;105(4):422-35.1 Ferreira FT et al. POC-CCA for diagnosing schistosomiasis364

11. Lambertucci JR, Drummond SC, Voieta I, Queiróz LC, Pereira PPN, Chaves BA, et al. An outbreak of acute Schistosoma mansoni schistosomiasis in a non-endemic area of Brazil: A report on 50 cases, including five with severe clinical manifestations. Clin Infect Dis. 2013;57(1):e1-6.

12. DeJonge N, Decaluw P, Hilberath GW, Krijger FW, Polderman AM, Deelder AM, Circulating anodic antigen levels in serum before and after chemotherapy with praziquantel in schistosomiasis mansoni. Trans $\mathrm{R}$ Soc Trop Med Hyg 1989; 83: 368-372.

13. Van Leishout L, Dejonge N, Mansour MM, Bassily S, Krijger FW, Deelder AM. Circulating cathodic antigen levels in serum and urine of schistosomiasis patients before and after chemotherapy with praziquantel. Trans R Soc Trop Med Hyg 1993; 87: 311-312.

14. Feldmeier H, Nogueira-queroz JA, Peixotoqueiroz MA, Doehring E, Dessant JP, De Alencar JE, et al. Detection and quantification of circulating antigen in schistosomiasis by monoclonal antibody. the quantification of circulating antigens in human schistosomiasis mansoni and haematobium: relation to the 
intensity of infection and disease status. Clin Exp Immunol 1986; 65: 232-243.

15. WHO (1998): Report of the WHO informal consultation on schistosomiasis control, Geneva WHO/ CDS/ CPC/ S1P/ 1999. 2.

16. Ahmed A. Yameny. The validity of the questionnaire to identify Schistosoma haematobium infection. J Biosci App Res .2017;3(4): 221228.

DOI: $10.21608 /$ JBAAR.2017.126468

17. Ahmed A. Yameny. The Validity of Haematuria and Proteinuria by chemical reagent strip for diagnosis of Schistosoma haematobium infection. J Biosci App Res .2017;3(4): 229236.

DOI: $10.21608 /$ JBAAR.2017.126469

18. WHO. 2007. Report of intercountry meeting on a strategy to eliminate schistosomiasis from the Eastern Mediterranean Region. Muscat, Oman.6-8 November.

19. Ahmed A. Yameny. The evolving schistosomiasis agenda 2017- 2020 in Egypt: Moving from control toward final elimination. J Biosci App Res .2017;3(2): 48- 54. DOI: $10.21608 /$ JBAAR.2017.124633

20. Eyong ME, Ikepeme EE, Ekanem EE. Relationship between Schistosoma haematobium infection and urinary tract infection among children in South Eastern, Nigeria. Niger Postgrad Med J2008;15:89-93.

21. Cheesbrough M. District laboratory practice in tropical countries part 2. United Kingdom: Cambridge University Press. Cambridge. 2006; 323-41.

22. Cheesbrough M. Laboratory-practice-intropical-countries-part-1. United Kingdom: Cambridge University Press. Cambridge. 2009;p.357

23. Ahmed A. Yameny. Evaluation of Nuclepore Filteration Technique for Diagnosis of Schistosoma haematobium Infection. J Biosci
App Res. 2015;1(6):335-341. DOI: $10.21608 /$ JBAAR.2015.106044

24. ElMorshedy H., Kinosien B., Barakat R., Omer E., Khamis N., Deelder A. M., et al. (1996) Circulating anodic antigen for detection of Schistosoma mansoni infection in Egyptian patients. Am. J. Trop. Med. Hyg. 54:149-153.

25. Polman K., Stelma F. F. Gryseels B., Van Dam G. J., Talla I., Niang M., et al. (1995) Epidemiological application of circulating antigen detection in a recent Schistosoma mansoni focus in northern Senegal. Am. J. Trop. Med. Hyg. 53:152-157.

26. Pearce, E.J., 2005. Priming of the immune response by schistosome egg. Parasite Immunol., 27: 265-270.

27. Armour, J., G.M. Urqhart and J.L. Ductan, 1997. Veterinary Parasitology. 2nd Edn., Blackwell Science Ltd., Oxford, New York, Pages: 64.

28. Demerdash ZA, Mohamed SH, Shaker ZA, Hassan SI, El attar GM, Saadel din AA, et al.Detection of circulating schistosome antigens in serum and urine of schistosomiasis patients and assessment of cure by a monoclonal antibody.J. Egypt. Soc. Parasitol.,25(2) 1995; 471 - 484.

29. Ndhlovu P, Cadman H, Gundersen S, Vennervald BJ, Friis H, Christensen NO.et al Circulating Anodic Antigen (CAA) levels in different age groups in a Zimbabwean rural community endemic for schistosoma haematobium determined using the magnetic beads Antigen- capture Enzyme-Linked Immunoassay. Am J Trop Med Hyg. 54(5),1996, pp. 537- 542.

30. Mahfouz A., Mahana N., Rabee I.and El Amir A., 2012. Evaluation of Different Immunological Techniques for Diagnosis of Schistosomiasis haematobium in Egypt. Biotechnology, 11: 10-19. 